\title{
Tennis elbow: an ultrasonographic study in tennis players
}

\author{
N. Maffulli, $\mathrm{MD}^{1}$, R. Regine, $\mathrm{MD}^{2}$, F. Carrillo, $\mathrm{MD}^{3}$, G. Capasso, $\mathrm{MD}^{4}$ and S. Minelli, $\mathrm{MD}^{5}$ \\ 1,4 University of Naples, First Medical School, First Institute of Orthopaedics and Traumatology, Naples, Italy \\ 2,3,5 University of Naples, Second Medical School, First Institute of Radiology, Naples, Italy \\ ${ }^{1}$ University of London, Institute of Child Health, Respiratory and Anaesthetic Unit, Sports Medicine Laboratory \\ and University of Naples, First Medical School, Institute of Physiology, Section of Sports Physiopathology
}

\begin{abstract}
The findings of ultrasound examination at and around the lateral humeral epicondyle in 41 tennis players suffering from so called tennis elbow are reported. Ultrasound examinations were performed with a real time ultrasound machine. The tenderness and functional impairment of tennis elbow may be caused by several different lesions, at times appearing in association. Six ultrasonographic characteristics could be identified:
\end{abstract}

Enthesiopathy The proximal part of the tendon was enlarged and there were echogenicity alterations.

Tendonitis The tendon of the extensor carpi radialis brevis was enlarged and areas of dyshomogeneous hypoechogenicity were evident with loss of the normal microscopic waveform structure of the tendon collagen.

Peritendonitis A thickening of the peritendonous lining was present.

Bursitis A bursa was located on the inferior surface of the tendon of the extensor carpi radialis brevis. Intramuscular haematoma Some circular or ovoid hypoechogenic areas within the muscular substance of the extensor carpi radialis brevis were evident. Mixed lesions These were not correlated with the intensity and the duration of the symptoms.

Ultrasonographic examination gives a detailed iamge of the structures involved in the tennis elbow syndrome, confirms the diagnosis, and may be useful in monitoring treatment.

Keywords: Tennis elbow, sports injuries, ultrasonography

\section{Introduction}

The term 'tennis elbow' (TE) refers to a painful condition at or around the lateral epicondyle of the humerus and the common extensor origin. It may radiate along the forearm. There is also localized tenderness over these areas, and pain on resisted active extension of the wrist when the elbow is fully extended ${ }^{1}$.

Although chronic overuse is said to cause lateral epicondylitis, its aetiology is still not well defined ${ }^{2}$, and several factors have been proposed. Some

Address for correspondence: N. Maffulli, Institute of Child Health, Sports Medicine Laboratory, 30 Guildford Street, London WC1N 1EH, UK

(C) 1990 Butterworth-Heinemann Ltd

0306-4179/90/030151-05 authors have suggested that enthesiopathy may be the unifying cause ${ }^{3}$, although several other lesions have been reported ${ }^{4}$. Some neurological syndromes, namely cervical spine disease with radiculopathy ${ }^{5}$ and posterior interosseus nerve entrapment ${ }^{6}$, may mimic the tennis elbow syndrome. Bursae have also been associated with $\mathrm{TE}^{7}$.

The factors involved in the aetiology of tennis elbow have been widely discussed ${ }^{8}$. On a purely anatomical basis, they include detachment from the lateral epicondyle of muscular or tendinous fibres of the extensor carpi radialis brevis during wrist movements, and impingement of bursae or synovial folds between the fibres of the extensor carpi radialis brevis and the elbow joint capsule 9 . A blood flow disturbance, causing relative ischemia and degeneration of the soft tissues around the lateral epicondyle, has also been proposed as a possible cause ${ }^{9}$.

Recent studies have shown that up to one third of American local league tennis players may suffer from tennis elbow ${ }^{10}$. Only a minority recover fully with medical treatment ${ }^{11}$. Causes indicate racquet factors, hitting technique, timing, and condition of the court. High frequency of play is the main contributory factor $^{10,11}$.

We report the findings of ultrasound examination in 41 tennis players suffering from TE.

\section{Patients and methods}

The series presented consists of 41 tennis players (24.3 \pm 7.3 years, range 16 to 36 years), only two were female. All were experienced, training three to five times a week and competing at least at regional level in their respective age groups for the year before the study. They had all presented with pain on the lateral aspect of the lateral humeral epicondyle and/or the common extensor origin for an average of 2.2 months (range 17 days to 9.8 months) before the study. About one third (15) had already sought medical advice with no beneficial effect. Neurological involvement and rheumatic diseases had been excluded in all patients, and a definite diagnosis of TE was confirmed independently by both the orthopaedic surgeons involved in the study.

All ultrasound examinations were performed using a real time ultrasound machine, equipped with a 
linear $7 \mathrm{MHz}$ probe, and a sector $5 \mathrm{MHz}$ probe. Ultrasound scan was performed by one of the radiologists, ensuring that the scanning beam was perpendicular to the structures studied ${ }^{12}$. The analysis of the scan was made on images by two other radiologists who had no prior knowledge of the patient whose image they were examining.

Eight patients were followed up to complete clinical resolution.

\section{Results}

Analysis of the ultrasound scan images confirmed the multiplicity of lesions associated with this clinical syndrome.

Enthesiopathy (five cases) The proximal part of the extensor carpi radialis brevis (ECRB) tendon was enlarged and there were echogenicity alterations.

Tendonitis (15 cases) The main tendon of ECRP was enlarged and areas of dishomogeneous hypoechogenicity were evident together with loss of the normal waveform structure of the tendon (Figure 1).

Peritenonitis (four cases) A thickening of the peritendonous lining was present. In one case, this was accompanied by an enlargement of the tendon itself but it was still possible to identify its normal structure.

Bursitis (five cases) An ovoid, well defined, hypoechoich area, a bursa, was located on the inferior surface of the tendon of the extensor carpi radialis brevis. It displaces the tendon dorsally (Figure 2).

Intramuscular haematoma (two cases) Some circular or ovoid hypoechogenic areas within the muscular substance of the ECRB, of different echogenicity from the muscle and ill-defined borders, were evident about one centimetre from the extensor carpi radialis attachment (Figure 3). Mixed lesions (seven cases) They were not correlated with the intensity and the duration of the symptoms.
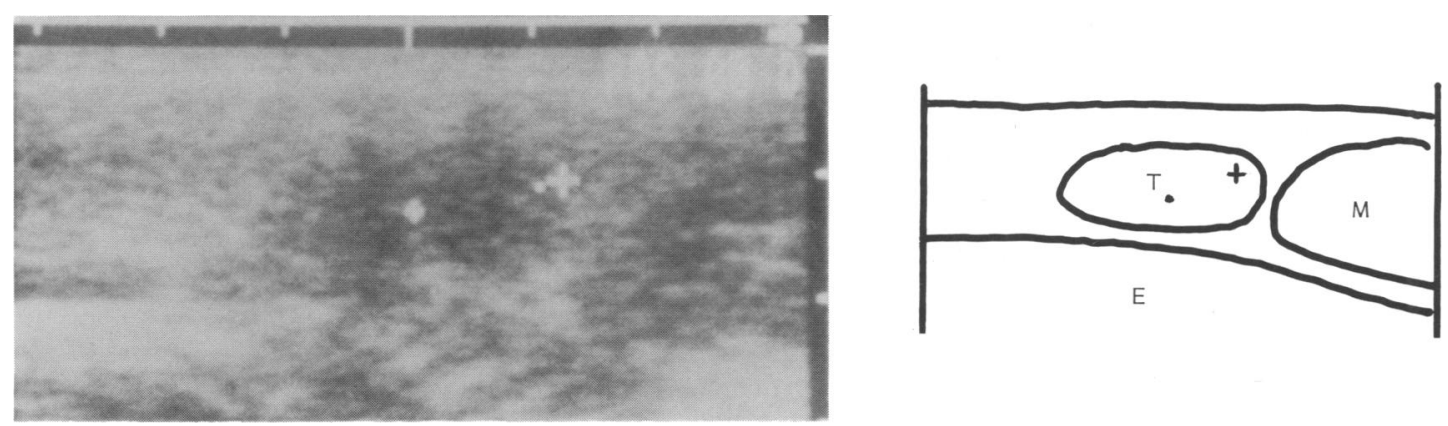

Figure 1. Tendonitis The tendon of the extensor carpi radialis brevis is enlarged and some areas of ultrasonic dyshomogeneity are present. The normal waveform structure is lost
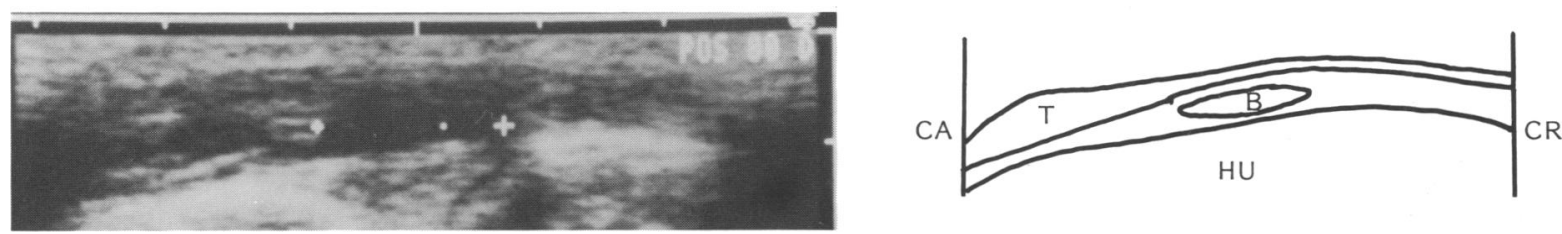

Figure 2. Bursitis A bursa is evident under the inferior aspect of the tendon of the extensor carpi radialis brevis, which is displaced dorsally
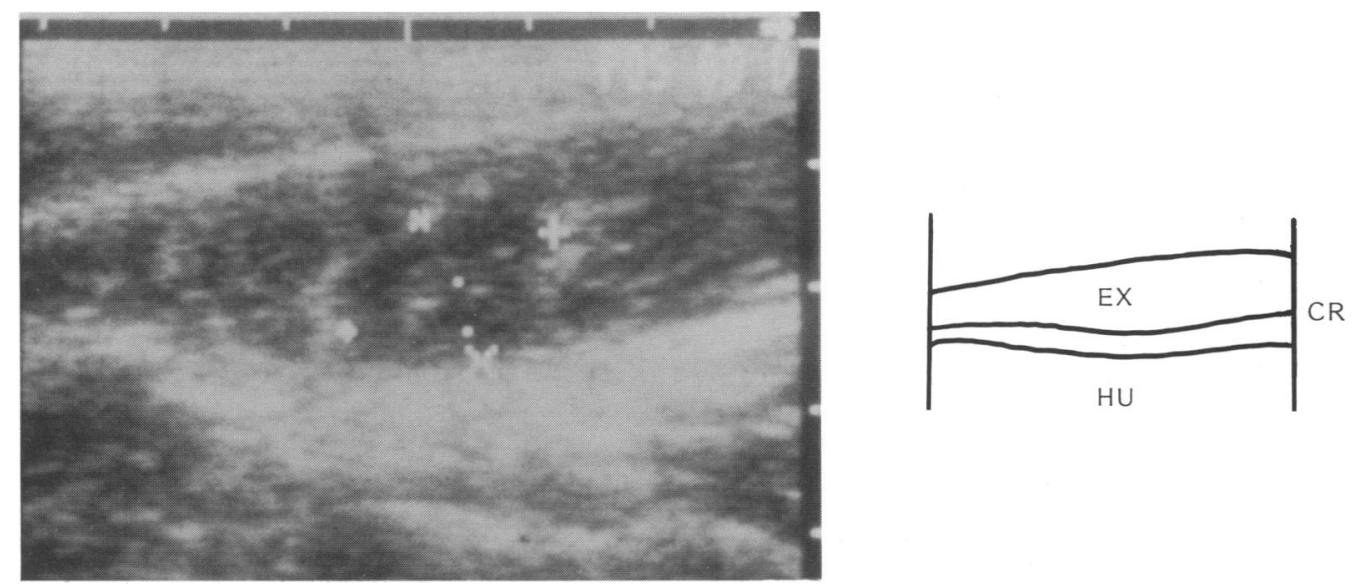

Figure 3. Intramuscular haematoma An ovoid area of hypoechogenic appearance, located within the muscular substance of the extensor carpi radialis brevis, about one centimetre from its attachment, is shown 

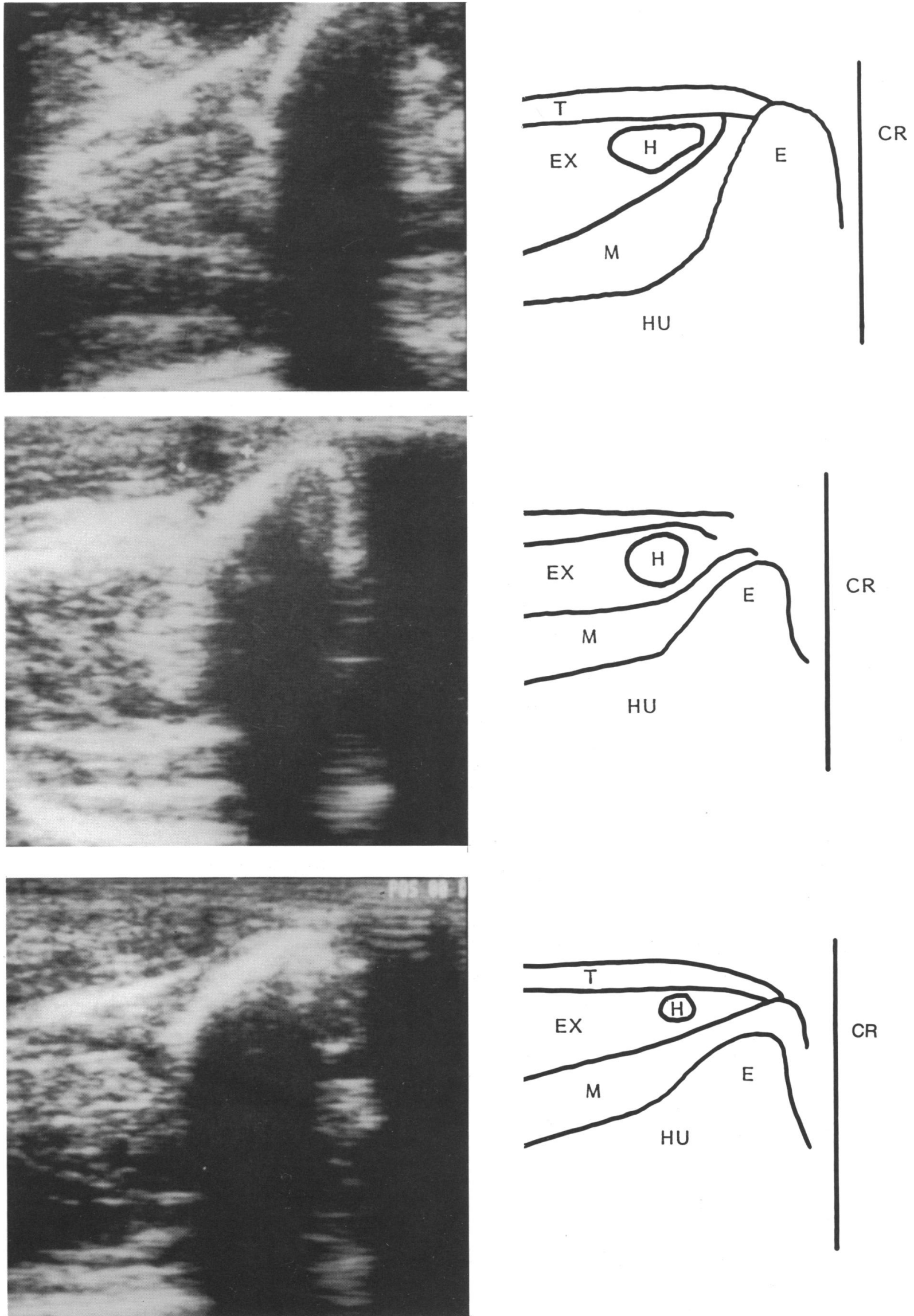

Figure 4. Evolution of an intramuscular haematoma treated conservatively Top: A Large ovoid hypoechoic mass is evident just distal to the musculo-tendinous junction. Middle: The mass is reduced in size after two months. Bottom: Four months after the first scan, there is still some ultrasonic evidence of the original intra-muscular haematoma, but the patient was fully asymptomatic 
No ultrasonographic abnormalities (three cases) This may have been due either to the acuteness of the lesions or their mildness or technical factors causing them to be beyond the resolution of the scanner used.

In 14 cases the tendon of ECRB appeared as a distinct anatomical structure ${ }^{9}$. (Two patients with an ultrasonographic picture of enthesiopathy, six with tendonitis, two with peritendonitis, one with bursitis, two with mixed lesions and one with no detectable ultrasonographic alterations.)

\section{Discussion}

The common extensor origin is classically described as a tendinous mass ${ }^{13}$. However, in accordance with other studies ${ }^{9}$, we found that the tendon of the extensor carpi radialis brevis could be a distinct structure. The fact that the surface of attachment is thus reduced may account for the decreased ability to sustain repeated stresses, and may thus be a predisposing factor for overuse injuries.

The pain evoked by movements and the localized tenderness can be explained by all the findings of this study. Typically, the symptoms are reproduced when the elbow is fully extended with the wrist dorsiflexed, and a downward force is applied on the middle finger with the patient resisting it $^{14}$, and in the last $20^{\circ}$ of extension when the forearm is pronated and the wrist is extended from a fully flexed position ${ }^{8}$. In these forced movements, the muscle either compresses the underlying inflamed bursa, or, being incapable of further lengthening, muscle or tendon micro-tears are produced.

At present, it is difficult to know whether ultrasonographic findings can be used as a prognostic means. In cases of definite bursitis, local steroid infiltration was prescribed, while muscle tears were treated conservatively with rest, stretching, oral NSAIDs and ultrasound therapy.

Three cases of long-standing and recurrent TE with an ultrasound diagnosis of tendonitis were treated surgically with multiple incisions along the longitudinal axis of the tendon. One further case, ultrasonographically diagnosed as an enthesiopathy, was treated with percutaneous release of the epicondylar muscle origin ${ }^{15}$ with full recovery. All the patients operated on are currently playing and regard themselves as fully healed.

Up to now, it is unclear how ultrasonographic findings may influence the management of TE, but a tentative classification of the underlying anatomical basis of the condition may be attempted.

\section{Key to illustrations}

B bursa; CA caudal; CR, cranial; E, lateral humeral epicondyle; EX, extensor carpi radialis brevis; $\mathrm{H}$, haematoma; HU, humerus; $M$, other muscles; $T$, tendon of the extensor carpi radialis brevis, or common extensor muscles tendinous mass

\section{Extra-tendinous alterations}

Muscular tears Haematoma of circular or ovoid appearance, hypoechogenic.

Bursitis Cavity lying just below the extensor carpi radialis brevis, adjacent to its radial head.

\section{Pathology of the tendon ${ }^{16}$}

Enthesiopathy Enlargement of the proximal part of the tendon and echogenicity alterations.

Tendonitis Enlargement and alterations of the substance of the tendon, possibly with inflammatory and degenerative appearances.

Peritendonitis Thickening of the tendon sheath all the way to the area of the muscle-tendon junction. A stenosing tenosynovitis may result ${ }^{17}$. Mixed lesions Any combination of the above mentioned pathologies may, at least theoretically, occur.

Until recently, although it was recognised that the variable sites of local tenderness could imply different pathology ${ }^{1}$, tennis elbow was considered a single entity $^{2}$. To our knowledge, this is the first in vivo study to show non-surgically that TE may be caused by several different pathological lesions.

Ultrasonographic examination gives a detailed image of the structures involved in the tennis elbow syndrome, confirms the diagnosis, and may be useful in monitoring treatment (Figure 4). Due to its safety, its contained costs, and the easiness of the scan itself, we believe that ultrasonography can become routine practice in the assessment of TE.

However, a potential limitation of the technique is the availability of high quality transducers, given that the axial resolving power of a $7.5 \mathrm{MHz}$ transducer is around five $\mathrm{mm}$. In our hands, this has allowed us to detect intramuscular haematomas of about two $\mathrm{mm}$ in diameter, and to study tendinous lesions in detail $^{18}$, being able to follow the evolution of post-traumatic haematoma in ruptured tendons ${ }^{19}$, and the image of single-thread suture in repaired tendons ${ }^{20}$.

\section{References}

1 Chard, M.D. and Hazleman, B.L. Tennis elbow - a reappraisal $\mathrm{Br} J$ Rheumatol 1989, 28, 186-190

2 Binder, A., Parr, G., Page Thomas, P. and Hazleman, B. A clinical and thermographic study of lateral epicondylitis Brit J Rheum 1983, 22, 77-81

3 Niepal, G.A. and Sitaj, S. Enthesopathy Clin Rheum Dis 1979, 5, 857-872

4 Friedlander, H.L., Reid, R.L. and Cape, R.I. Tennis elbow Clin Orthop 1967, 51, 109-116

5 Murray-Leslie, C.F. and Wright, V. Carpal tunnel syndrome, humeral epicondylitis and the cervical spine. A study of clinical and dimensional relations $\mathrm{Br}$ Med J 1976, 1, 1439-1442

6 Werner, C. Lateral elbow pain and posterior interosseus nerve entrapment Acta Orthop Scand 1979, Suppl, 174, 16-2

7 Bosworth, D.M. The role of the orbicular ligament in tennis elbow J Bone Joint Surg 1955, 37A, 527-533

8 Nirschl, R.P. Tennis elbow Orthop Clin North Am 1972, 4, 787-800 
9 Briggs, C.A. and Elliot, B.G. Lateral epicondylitis. A review of the structures associated with tennis elbow Anat Clin 1985, 7, 149-153

10 Legwold, G. Tennis elbow: joint resolution by conservative treatment and improved technique Phys Sportsmed 1984, 12, 168-182

11 Carrol, R. Tennis elbow: incidence in local league players Br J Sports Med 1981, 15, 250-256

12 Fornage, B.D. The hypoechoic normal tendon. A pitfall J Ultrasound Med 1987, 6, 19-22

13 Last, R.J. 'Anatomy. Regional and Applied' Churchill Livingstone, Edinburgh, 1984

14 Kulund, D.N. 'The Injured Athelete' J.B. Lippincott Company, Philadelphia, 1982

15 Baumgard, S.H. and Schwartz, D.R. Percutaneous release of the epicondylar muscles for humeral epicondylitis Am J Sports Med 1982, 10, 233-236
16 Puddu, G., Ippolito, E. and Postacchini, F. A classification of Achilles tendon disease Am J Sports Med 1976, 4, 145-149

17 Viikari-Juntura, E. Tenosynovitis, peritendinitis and the tennis elbow syndrome Scan J Work Environ Health 1984, 10, 443-449

18 Maffulli, N., Regine, R., Angelillo, M., Capasso, G. and Filice, $S$. Ultrasound diagnosis of Achilles tendon pathology in runners $B r J$ Sports Med 1987, 21, 158-162

19 Maffulli, N., Dymond, N.P. and Capasso, G. Ultrasonographic findings in subcutaneous rupture of Achilles tendon J Sports Med Phys Fit 989, 29, 365-368

20 Maffulli, N., Dymond, N.P. and Regine, R. Surgical repair of ruptured Achilles tendon in sportsmen and sedentary patients: a longitudinal ultrasound assessment Int J Sports Med 1990, 11, 78-84

\section{Royal College of Surgeons of England Porritt Fellowship}

Applications are invited for the 1991 aware of the Porritt Fellowship given by the Winthrop Foundation in honour of the Rt Hon The Lord Porritt GCMG CGVO CBE FRCS, to encourage the study of sports medicine and injuries in sport.

The purpose of the Porritt Fellowship is to encourage study of the physiology and biochemistry of sporting achievement and/or the aetiology, pathology, prevention and treatment of accidents and injuries associated with sporting activities. The work may be carried out in the UK or abroad, and should be suitable for publication or form part of a thesis for higher qualifications.

A candidate must hold the Fellowship of a Royal College of Surgeons in Great Britain or Ireland, or of one of their Faculties or be a medically qualified member of the staff of one of their scientific departments. Successful candidates will probably hold university, NHS or other salaried posts, and will use their Fellowships to travel to centres at home or abroad to study.

The value of the Fellowship is $£ 5,000$ tenable normally for a period of one year, from September 1991, and a Porritt Fellow may be invited to deliver a Porritt Lecture by the British Association of Sport and Medicine.

Applications must reach R.H.E. Duffett not later than Friday 26th April 1991 and must include the following:

- Curriculum vitae

- Statement (maximum 1000 words) of the study or research project, including the location and facilities already available for the project

- Particulars of the applicant's salary and of any additional financial support promised or applied for

- Names and addresses of two referees, one of whom should be the head of the department in which the applicant is working.

Applications should be made to: RHE Duffett, MA, Secretary, The Royal College of Surgeons of England, 35/43 Lincoln's Inn Fields, London WC2A 3PN, UK. 